\title{
Behavioral measures of cortical hyperexcitability assessed in people who experience visual snow
}

Allison M. McKendrick, $\mathrm{PhD}$

Yu Man Chan, $\mathrm{PhD}$

Melissa Tien, MMed (Ophthalmology),

FMAS

(Ophthalmology)

Lynette Millist, BAppSci

Meaghan Clough, PhD

Heather Mack, MBBS,

$\mathrm{PhD}$, RANZCO

Joanne Fielding, $\mathrm{PhD}$

Owen B. White, MD, $\mathrm{PhD}$

Correspondence to

Dr. McKendrick:

allisonm@unimelb.edu.au

\section{ABSTRACT}

Objective: To determine whether visual perceptual measures in people who experience visual snow are consistent with an imbalance between inhibition and excitation in visual cortex.

Methods: Sixteen patients with visual snow and 18 controls participated. Four visual tasks were included: center-surround contrast matching, luminance increment detection in noise, and global form and global motion coherence thresholds. Neuronal architecture capable of encoding the luminance and contrast stimuli is present within primary visual cortex, whereas the extraction of global motion and form signals requires extrastriate processing. All these tasks have been used previously to investigate the balance between inhibition and excitation within the visual system in both healthy and diseased states.

Results: The visual snow group demonstrated reduced center-surround contrast suppression ( $p=$ 0.03 ) and elevated luminance increment thresholds in noise $(p=0.02)$. Groups did not differ on the global form or global motion task.

Conclusion: Our study demonstrates that visual perceptual measures involving the suprathreshold processing of contrast and luminance are abnormal in a group of individuals with visual snow. Our data are consistent with elevated excitability in primary visual cortex; however, further research is required to provide more direct evidence for this proposed mechanism. The ability to measure perceptual differences in visual snow reveals promise for the future development of clinical tests to assist in visual snow diagnosis and possibly a method for quantitatively assaying any benefits of treatments. Neurology ${ }^{\circledR}$ 2017;88:1243-1249

\section{GLOSSARY}

RM-ANOVA = repeated-measures analysis of variance.

Visual snow is an uncommon neurologic disorder where people perceive persistent static-like "snow" 1,2 consistent with aberrant neuronal firing in visual cortex. We measure visual performance in people with visual snow using tasks that assay the balance between inhibition and excitation. Such tasks have been used to explore other conditions where the balance between inhibition and excitation is relevant: for example, migraine ${ }^{3-6}$ and epilepsy. ${ }^{7}$ We included 2 tasks assessing relatively primitive visual features encoded early in visual processing: luminance increment detection in noise ${ }^{8,9}$ and contrast suppression. ${ }^{3,10}$ We included a further 2 tasks (global form and motion) where the neuronal architecture required to extract the relevant stimulus features is not available until extrastriate cortex. ${ }^{11-15}$

We test the hypothesis that people with visual snow demonstrate perceptual performance consistent with aberrant cortical excitability in both primary and extrastriate visual areas. Three of our tasks (luminance increment detection, global form, and motion) require the extraction of signal from explicit noise. For these, we hypothesized that performance would be impaired due to added spontaneous neural noise in the visual snow group. The contrast suppression $\operatorname{task}^{10}$ is considered a perceptual analogue of visual cortical center-surround circuitry. ${ }^{16}$ We predicted

From the Department of Optometry and Vision Sciences (A.M.M., Y.M.C.), the Department of Neurology, Royal Melbourne Hospital (M.T., L M., M.C., J.F., O.B.W.), and the Faculty of Medicine, Dentistry and Health Sciences, and Department of Surgery (H.M.), The University of Melbourne; School of Psychological Sciences (M.C., J.F.), Monash Institute of Cognitive and Clinical Neurosciences, Monash University; and Department of Ophthalmology (H.M.), Royal Melbourne Hospital, Australia.

Go to Neurology.org for full disclosures. Funding information and disclosures deemed relevant by the authors, if any, are provided at the end of the article. 
that the visual snow group would show weaker contrast suppression (less inhibition) resulting in a shifted perceptual matching point rather than worse performance. Inclusion of this task enables the disentanglement of altered perception from generalized poorer performance. To our knowledge, ours is the first study to assess perceptual indications of cortical hyperexcitability in individuals with visual snow.

METHODS Participants. Sixteen patients with visual snow (mean age 28 years [17-51 years], mean snow duration 8.8 years [0.3-40 years], 9 female, 7 male) participated in this study. Eighteen healthy adults (mean age 29 years [18-56 years], 10 female, 8 male) with no history of neurologic or psychiatric disorders served as the control group. Visual snow patients were recruited from the Royal Melbourne Hospital, The Malvern Neurology Group at Cabrini Medical Centre, and the Ophthalmology Clinic at the Eye Surgery Associates in Australia. Controls were recruited from the community. Recruitment and data collection was conducted between January 2016 and April 2016. Sample size was estimated from previous studies conducted in people with migraine on a similar set of psychophysical tasks. ${ }^{3,6,8}$

A summary of the demographics and visual and nonvisual symptoms of the visual snow group appears in the table. Visual snow features were considered with reference to recently proposed diagnostic criteria, ${ }^{2}$ including (1) dynamic, continuous visual snow for more than 3 months; (2) at least 2 additional visual symptoms of palinopsia, entoptic phenomena, photophobia, or nyctalopia; (3) symptoms not consistent with typical migraine visual aura; and (4) symptoms not better explained by any other disorder or intake of psychotropic drugs. Three admitted to a history of psychogenic drug use (marijuana and ecstasy) but their visual snow symptomatology was not directly related in timing to that history. All underwent a full ophthalmic assessment to establish normal vision, color vision (Ishihara color plates), and normal retinal anatomy and function via dilated fundus slit-lamp examination and normal full-field electroretinography, in accordance with the International Society for Clinical Electrophysiology of Vision standards. ${ }^{17}$ All participants had best-corrected visual acuity of 6/7.5 or better. Exclusion criteria for controls were a history of migraine with or without aura, a history of visual and neurologic conditions, and consumption of medication known to affect visual and cognitive function.

Standard protocol approvals, registrations, and patient consents. Ethics approval was granted by the Melbourne Health Human Research Ethics Committee. All participants provided written informed consent in agreement with the Declaration of Helsinki prior to participation.

Equipment. Stimuli were generated with a ViSaGe system (Cambridge Research Systems, Ltd., Kent, UK) using customized software written in MatLab version 7.6.0.324 (R2008a) (Mathworks, Natick, MA) and presented on a CRT monitor (100 $\mathrm{cd} / \mathrm{m}^{2}$, frame rate: $100 \mathrm{~Hz} ; 1,024 \times 768$ pixels; Sony Trinitron G520, Tokyo, Japan). Observers viewed the stimuli binocularly via a chinrest from a distance of $83 \mathrm{~cm}$ and responded using a button box (CB6; Cambridge Research Systems).

Stimuli and procedures. All participants completed all 4 tasks. To minimize bias due to fatigue or learning, task order was randomized between observers. Observers completed practice trials until they were confident with the requirements. The total test duration lasted no more than 40 minutes, including rest breaks when required.

Table Patient demographics and visual and nonvisual symptoms

\begin{tabular}{|c|c|c|c|c|c|c|c|c|c|c|}
\hline $1^{a}$ & 18 & $\mathrm{~F}$ & 18 & 0.3 & + & + & + & + & + & + \\
\hline 3 & 21 & $\mathrm{~F}$ & 17 & 4 & + & + & + & + & - & + \\
\hline 4 & 32 & $\mathrm{~F}$ & 31 & 1 & + & + & + & + & + & + \\
\hline 5 & 17 & $M$ & 10 & 7 & + & + & + & + & - & + \\
\hline 9 & 17 & $M$ & 14.5 & 2.5 & + & - & + & + & - & + \\
\hline 10 & 51 & $M$ & 11 & 40 & - & - & + & - & - & - \\
\hline 11 & 18 & $M$ & 4 & 14 & + & + & + & + & + & + \\
\hline 12 & 32 & $\mathrm{~F}$ & 12 & 20 & + & - & + & - & + & + \\
\hline 13 & 24 & $M$ & 23 & 1 & + & + & + & + & + & + \\
\hline 14 & 37 & $\mathrm{~F}$ & 33 & 4 & + & + & + & + & + & + \\
\hline
\end{tabular}

${ }^{a}$ History of hallucinogenic drug. 
Luminance increment detection in noise. Figure $1 \mathrm{~A}$ illustrates the luminance increment detection task. Each presentation interval $(500 \mathrm{~ms})$ presented 2 squares $(4 \times 4$ degrees $)$ filled with luminance noise pixels presented on the left and right of the screen with a center-to-center distance of 7.2 degrees. One of the squares also contained a 1.5-degree diameter circular luminance increment. The luminance of the noise pixels was randomly sampled from a Gaussian distribution with a mean luminance of $50 \mathrm{~cd} / \mathrm{m}^{2}$ and SD of 0.125 (low noise) or 0.25 (high noise) of the mean. We included 2 levels of background noise to determine whether patients with visual snow are more affected by higher noise levels than controls, consistent with an accelerating elevation in aberrant neural firing that is multiplicative with background stimulation level. ${ }^{9}$ The observers' task was to identify which square (left or right) contained the luminance increment. Luminance increment detection threshold was estimated using 2 interleaved 3-up-1-down staircases of 6 reversals. Three correct responses resulted in the luminance increment being decreased by $20 \%$, whereas each incorrect response increased the increment by $20 \%$. Thresholds were calculated as the mean of the last 4 reversals of each staircase with the grand mean being the average of the 2 staircases.

Figure 1 Illustration of the visual stimuli

A. Luminance noise

High noise

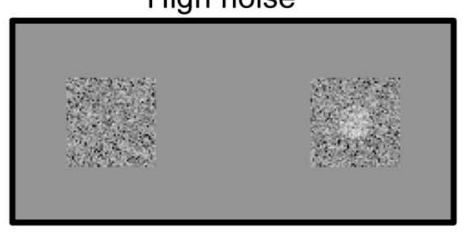

Presentation interval (500 ms)

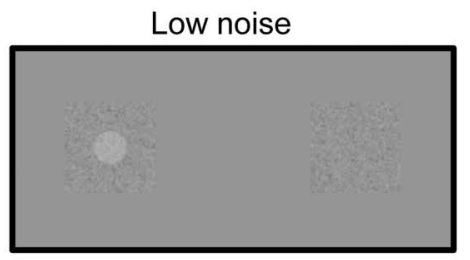

Presentation interval $(500 \mathrm{~ms})$

Which square contains the luminance increment circle?

\section{Global form}

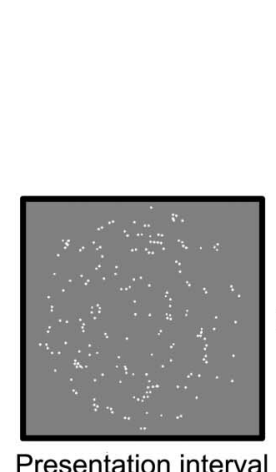

(500 ms)

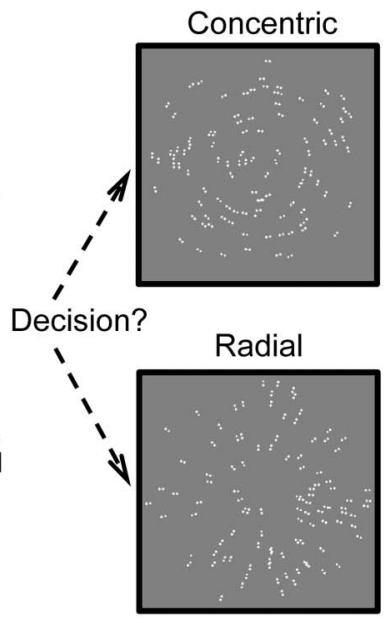

Do the dots appear to be arranged in a concentric or radial pattern?

\section{B. Center-surround}

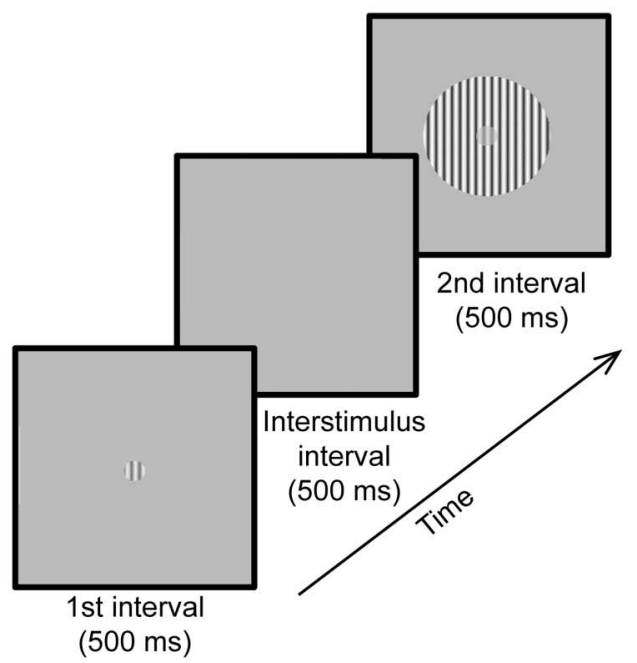

Which stimulus contains a central area at a higher contrast?

\section{Global motion}

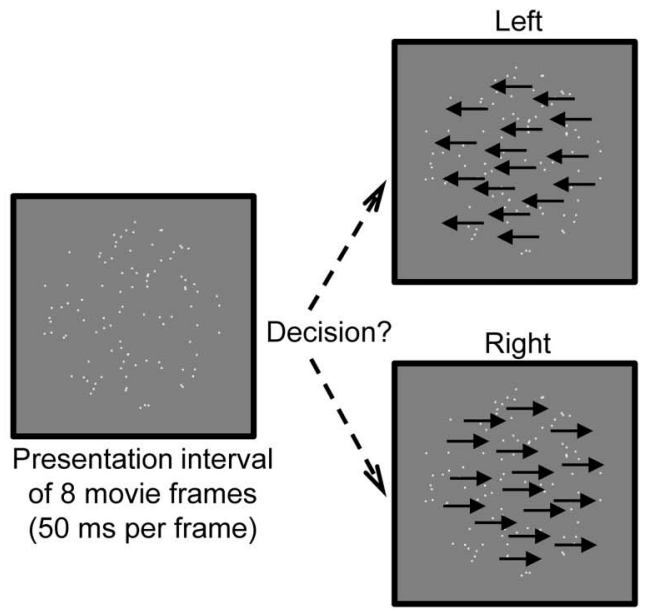

Do the dots appear to move toward the left or right?

(A) Illustration of the luminance increment detection in noise stimuli. (B) Illustration of the center surround task. The reference stimulus in the 2 nd interval had a $95 \%$ surround contrast and a $40 \%$ central contrast. (C) Illustration of the global form task. The dots were arranged in either a concentric or a radial pattern. (D) Illustration of the global dot motion task. For each set of movie frames, a proportion of the dots were randomly chosen to contain a local motion towards the specified global motion while the remaining proportion of dots moved in random directions. 
Center-surround contrast matching task. The contrast matching task was based on those used in other experiments exploring proposed imbalance between inhibition and excitation (including in migraine, ${ }^{3}$ schizophraenia, ${ }^{18}$ and healthy normal aging ${ }^{19-21}$ ). Participants viewed a central circular patch of sinusoidal grating of variable contrast (radius 0.67 degrees, spatial frequency $=2$ cycles/ degree, drifting at 2 degrees/s, vertical orientation) in the first interval (500 ms) followed by the reference patch (contrast of 40\%) with or without an annular surround ( $0 \%$ or $95 \%$ contrast, 4 degrees diameter) in the second interval (500 ms) (figure 1B). Participants responded whether the central grating patch was of higher contrast in the first or second interval. The task measured the perceived contrast to match the central patch, when on its own (to test the ability make matched contrast judgments) and when surrounded by the higher contrast annulus (which typically suppresses perceived contrast). A 2-interval-forced-choice design was employed with 2 interleaved 3-up-1-down staircases with a step size of 20\%, which terminated after 6 reversals. Individual perceived contrast threshold was taken as the grand mean of the last 4 reversals of the 2 staircases. For each observer, the difference between the perceived contrast of the test patch in the with-surround and the without-surround test condition was calculated as an estimate of center-surround suppression.

Global form stimuli. Figure 1C illustrates the global form task. The stimuli were Glass patterns ${ }^{22}$ constructed of 100 pairs of white dots $\left(8.6 \mathrm{arc}\right.$ min diameter, $90 \mathrm{~cd} / \mathrm{m}^{2}$ ) on a gray background (half of max luminance $=50 \mathrm{~cd} / \mathrm{m}^{2}$ ) presented for $500 \mathrm{~ms}$. Dot pairs had a center-to-center separation of 24 minutes of arc, and were placed within a 10-degree circular aperture. A percentage of the dot pairs were arranged in the signal orientation, with the remainder being noise pairs. Signal pairs were randomly positioned with dipole coordinates oriented at 0 (radial global form) or 90 degrees (concentric global form) on each trial. Noise pairs were positioned with random orientations. Participants responded whether the presented pattern was radial or concentric in form. Coherence thresholds (percentage of signal pairs) were determined using 2 interleaved staircases. For each staircase, 3 correct responses resulted in an increase in the percentage of noise dipoles, and each incorrect response decreased the percentage of noise dipoles. Each staircase terminated at the end of 6 reversals with an initial step size of $8 \%$ coherence, followed by $4 \%$ in the second reversal and $2 \%$ for the final 4 reversals.

Global dot motion stimuli. Figure 1D schematically depicts 1 of 8 movie frames of the global dot motion stimuli. One hundred white dots $\left(90 \mathrm{~cd} / \mathrm{m}^{2}\right)$ were presented within a $10^{\circ}$ aperture on a gray background $\left(50 \mathrm{~cd} / \mathrm{m}^{2}\right)$. Dots were 8.6 arc $\mathrm{min}$ in diameter. In the first movie frame, dots were randomly placed within the aperture. A further 7 frames were generated with each shown for 50 ms. For each frame, dots were chosen to be either signal (redrawn in the coherence direction to result in a dot velocity of 2.86 degrees $/ \mathrm{s}$ ) or noise (redrawn in a random direction except within 10 degrees of the signal direction). The signal direction was chosen to be right or left on each trial at random. If the displacement moved the dots outside the aperture, they were randomly positioned within the aperture in the next frame. The dots allocated to be signal dots were selected on each frame to minimize local motion cues. Observers had to choose whether the global motion direction was left or right. The motion coherence of the stimuli (percentage signal dots) was adjusted based on 2 interleaved 3-up-1-down staircases that terminated after 6 reversals. Both staircases varied with an initial step size of $8 \%$ coherence, followed by $4 \%$ in the second reversal and $2 \%$ for the final 4 reversals. Individual global dot motion coherence thresholds were taken as the mean of the last 4 reversals and the grand mean taken as the mean of the 2 staircases.

Analyses. Statistical analyses were conducted in SPSS Statistics version 21 (IBM, Armonk, NY). Groups were compared using
Mann-Whitney $U$ tests on the medians or a repeated-measures analysis of variance (RM-ANOVA) as appropriate.

RESULTS Luminance increment detection in noise. Figure 2 shows the luminance increment thresholds for both high and low noise conditions. Thresholds were elevated in both groups in the presence of higher luminance noise (RM-ANOVA: main effect of noise level: $\left.F_{1,32}=69.4, p<0.001\right)$. The patients had elevated thresholds for both noise conditions (main effect of group: $F_{1,32}=5.35, p=0.02$; no significant interaction between group and noise level: $F_{1,32}=$ 2.77, $p=0.11$ ).

Center-surround contrast matching. Figure $3 \mathrm{~A}$ shows the ability to contrast match the central patch in the absence of a surround. Both the patient and control groups were able to match the test patch reliably to its veridical contrast of $40 \%$ (figure $3 \mathrm{~A}$ ) (VS median = 40.64, control median $=40.15)$ (Mann Whitney $U=$ $143.5, p=0.772$ ). When the high-contrast surround was added, the patients with visual snow showed less contrast suppression than controls (higher contrast thresholds) (figure 3B) (VS median $=27.63$, control median $=32.84)(U=198, p=0.024)$. Panel $\mathrm{C}$ shows the data computed as a suppression index. This index was calculated as the difference between each individual's perceived contrast threshold in the presence and absence of the surround. Patients with visual snow had significantly reduced center-surround

Figure 2 Luminance increment detection thresholds measured in the presence of luminance noise

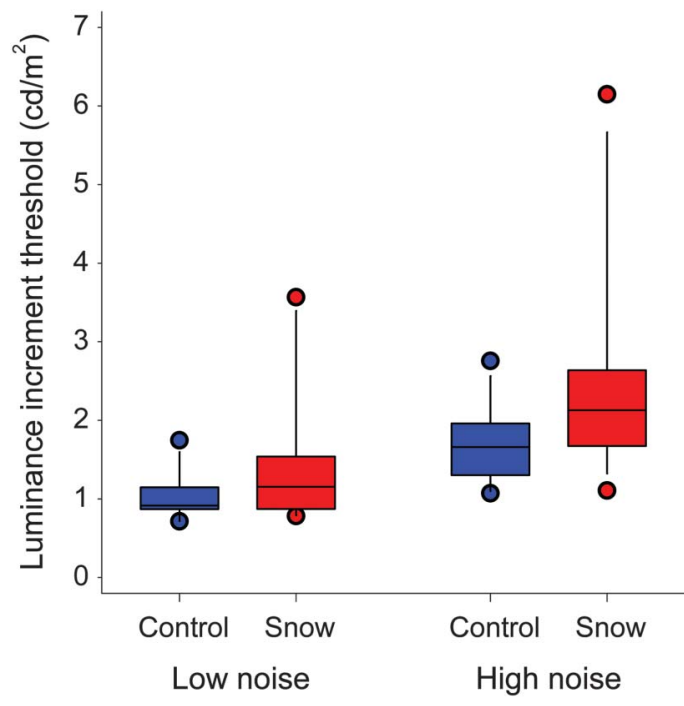

Patients with visual snow are shown as hatched and controls as shaded. Patients with visual snow had significantly higher increment detection thresholds in the presence of high noise but not in the presence of low noise. Boxes represent the median, 25th, and 75th percentiles with symbols showing the highest and lowest thresholds. 

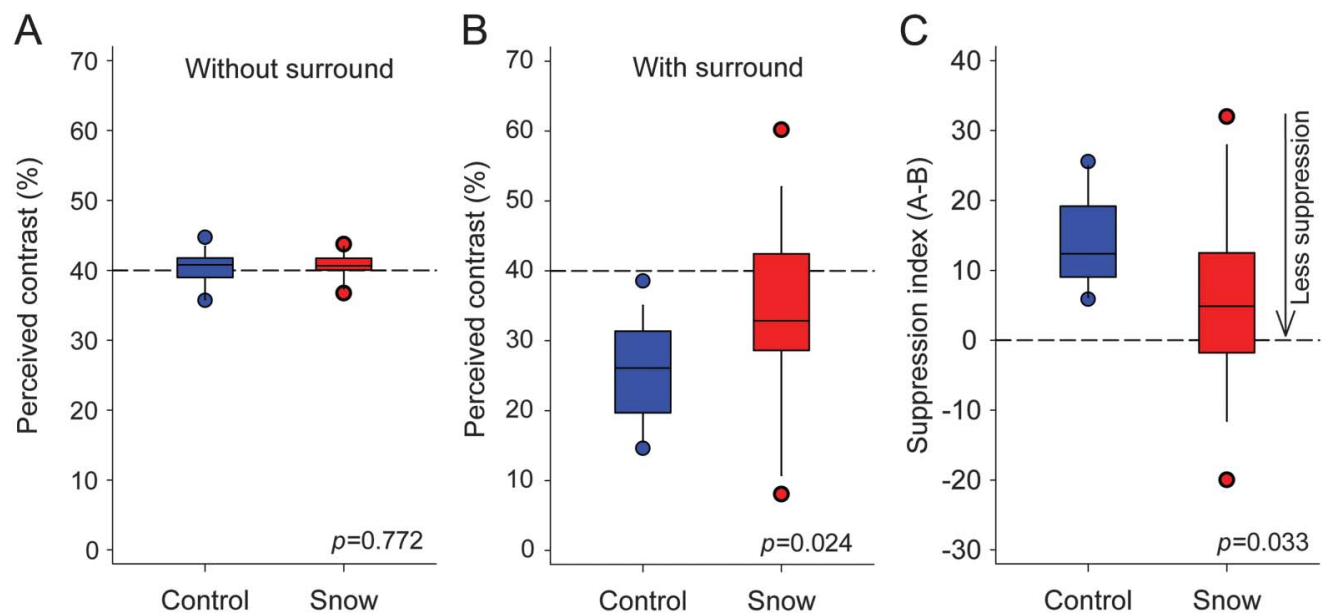

Patients with visual snow are shown as hatched and controls as shaded in the absence (A) and presence (B) of the high contrast surround. Boxes and whiskers are as for Figure 2. (C) Suppression indices were computed for each individual as the threshold difference between (A) and (B). The presence of the surround resulted in significantly less suppression of contrast for the patients with visual snow.

suppression than the control group (figure 3C) (VS median $=4.38$, control median $=11.70)(U=$ 76.0, $p=0.033$ ), with several patients showing facilitation rather than suppression.

Global form and global motion. There was no difference between groups for either the global form task (figure 4A: VS median $=28.25 \%$, control median $=26.00 \%$, $U=123.0, p=0.469$ ) or the global motion task (figure $4 \mathrm{~B}: \mathrm{VS}$ median $=13 \%$, control median $=14.4 \%, U=$ 129.5, $p=0.616$ ).

DISCUSSION Visual snow represents a challenge due to the diagnosis hitherto being entirely based on subjective patient reports, after exclusion of other pathology. Here we show that visual perception is altered in visual snow, in a fashion consistent with elevated spontaneous neural firing in visual cortex. $\mathrm{Pa}$ tients with visual snow showed, on average, an elevation in the luminance increment threshold required to detect a patch presented on luminance noise, and also showed on-average reduction in the level of contrast suppression induced by surrounding visual stimuli.

The elevated thresholds for luminance increment detection in the visual snow group were independent of background luminance noise level (figure 2). This

Figure 4 Global form and global motion coherence thresholds

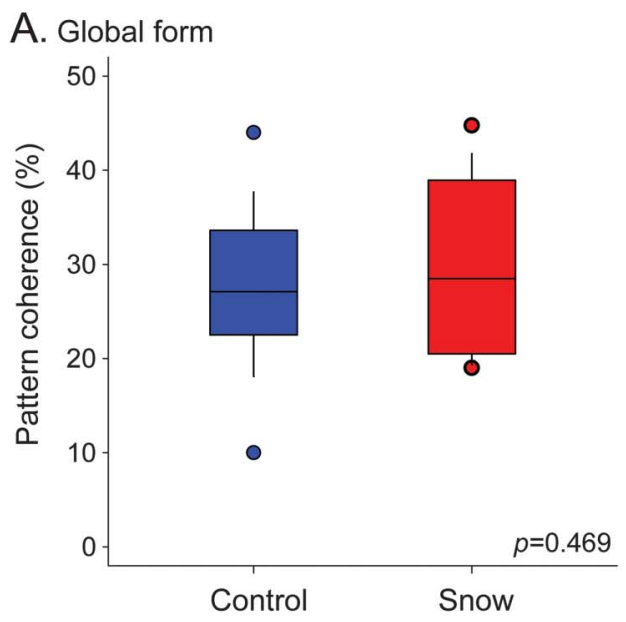

B. Global motion

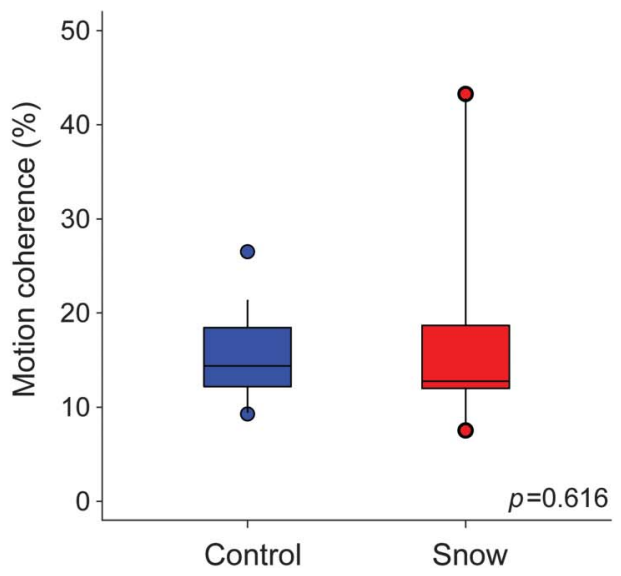

(A) Pattern coherence thresholds measured in the patients with visual snow (hatched) and controls (shaded) in the glass pattern discrimination task. Patients with visual snow did not perform significantly differently from the control group. (B) Motion coherence thresholds measured in the global dot motion discrimination task presented in the same format as in (A). Patients with visual snow did not perform significantly differently from the control group. Boxes and whiskers in same format as Figure 2. 
outcome is consistent with the subjective reports of many of the patients who stated that their visual snow static was present continuously rather than being tagged to specific visual environments. A different pattern of results is observed in individuals with migraine (without visual snow). Previous studies report that patients with migraine with aura and migraine without aura show elevated luminance increment thresholds that are multiplicative with background noise. ${ }^{8,9}$ Hence in migraine, performance is best explained by a model of increasing aberrant neural firing with increasing background visual stimulation. This contrasts with the visual snow outcomes where thresholds were elevated similarly in both noise conditions.

On average, the visual snow group demonstrated less surround suppression of perceived contrast (figure 3). Surround suppression of contrast is a well-studied phenomenon both from a perceptual and neurophysiologic perspective, most commonly at the level of primary visual cortex (for example, see references 16, 23-25). Centersurround contrast interactions in primary visual cortex arise from lateral inhibition, feedforward connections from lateral geniculate nucleus, and feedback from extrastriate areas. ${ }^{26,27}$ Our current data cannot disentangle these mechanisms, but further work that varies both stimulus factors, perhaps combined with neuroimaging, should assist in localizing the likely neural substrate with greater precision. Of note is the fact that the task involves the matching of perceived contrast. Consequently, anomalous results for this task do not imply poorer performance but instead indicate a difference in the subjective contrast appearance. Participants were naive to the expected perceptual effect. Consequently, unusual matching thresholds are not easily explicable by poor attention, visual aversion, or other nonvisual factors that are expected to result in elevated thresholds for all tasks.

There was no difference in performance between the visual snow and control groups for the global form and motion tasks. Previous studies have shown that performance on these tasks is, on average, impaired in people who experience migraine with or without aura. ${ }^{28-32}$ The absence of a difference in performance in our visual snow patients suggests that their visual anomalies are largely localized to luminance and contrast features, and that the building blocks for determining global form and motion (such as the encoding stimulus orientation and direction cues) are intact. Our data also do not support the concept of a generalized problem in noise exclusion, which appears to be a feature of some migraine cohorts. ${ }^{8,28}$

The sample size of this study was relatively small (16 individuals) and significant interindividual variability was noted. A significantly increased sample of visual snow patients would enable the addressing of further questions such as whether performance correlates with particular visual snow features. Interestingly, we did not find a difference in performance between those with comorbid migraine in our cohort (10 individuals) and those without ( 6 individuals): all comparisons were nonsignificant $(p>0.05)$. While this may reflect a sample size issue, it nevertheless suggests that the dominant feature influencing their performance on our visual tasks is their visual snow phenotype. We chose the tasks in this study in part because they had proved useful in revealing differences between migraine and nonheadache control groups when measured interictally. The pattern of experimental findings in our visual snow group was different from those typically observed in migraine. , $9,28,30^{\text {Now that we }}$ have identified differences in performance between visual snow group and control groups, and have shown that these differences do not share the same pattern that has been typically reported for people with migraine, a larger future study may be warranted to compare directly between visual snow with migraine, visual snow without migraine, and migraine alone.

We included 3 participants with a history of psychogenic drug use in our visual snow group. The recently proposed criteria for visual snow by Schankin et $\mathrm{al}^{2}$ exclude individuals where the visual snow might be explicable by intake of psychotropic drugs. These 3 individuals reported a history of marijuana and ecstasy use and could not be distinguished from the remainder of our visual snow cohort in terms of symptoms or performance; however, it is noted that the sample size is too small to identify subtle differences. Furthermore, 2 of these people had comorbid migraine. It is possible that the origin for cortical hyperexcitability in these 3 patients may be different than the remainder; however, the resulting symptomatic visual cortical hyperexcitability appears similar.

The scientific understanding of visual snow is in its infancy. Our study demonstrates that visual perceptual measures involving the suprathreshold processing of contrast and luminance are abnormal in a group of individuals with visual snow. Our data are consistent with elevated excitability in primary visual cortex; however, further research is required to provide more direct evidence for this hypothesis. Nevertheless, the ability to measure perceptual differences in visual snow reveals promise for the future development of clinical tests to assist in visual snow diagnosis and possibly an additional method for assaying benefits of treatments.

\section{AUTHOR CONTRIBUTIONS}

Allison M. McKendrick: study concept and design, analysis and interpretation, writing of manuscript, study supervision. Yu Man Chan: acquisition of data, analysis and interpretation, writing of manuscript. Melissa Tien: acquisition of data, critical revision of the manuscript for important intellectual content. Lynette Millist: acquisition of data, critical revision of the manuscript for important intellectual content. Meaghan Clough: acquisition of data, critical revision of the manuscript for important intellectual content. Heather Mack: acquisition of data, critical revision of the 
manuscript for important intellectual content. Joanne Fielding: study concept and design, interpretation of results, critical revision of the manuscript for important intellectual content. Owen B. White: study concept and design, interpretation of results, critical revision of the manuscript for important intellectual content, study supervision.

\section{STUDY FUNDING}

National Health and Medical Research Council Project Grant APP1081874 (to A.M.M.)

\section{DISCLOSURE}

A. McKendrick receives research support from Heidelberg Engineering GmbH (Germany), CenterVue PdA (Italy), and Haag-Streit AG (Switzerland), has research funding from the Australian Research Council and Australian National Health and Medical Research Council (APP1081874), and is an editorial board member of the following journals: Graefe's Archive of Clinical \& Experimental Ophthalmology, Clinical \& Experimental Optometry, and Translational Vision Science Technology. Y. Chan, M. Tien, L. Millist, M. Clough, and H. Mack report no disclosures relevant to the manuscript. J. Fielding receives research support from Novartis, Biogen, and Genzyme and from an Australian Research Council Linkage Grant (ID LP120200033) 2012-2015. O. White is an Associate Editor for Frontiers in Neuro-ophthalmology, receives research support from Novartis, Biogen, and Genzyme, and received honoraria for speaking from Biogen and Genzyme. Go to Neurology.org for full disclosures.

Received September 1, 2016. Accepted in final form January 6, 2017.

\section{REFERENCES}

1. Schankin CJ, Goadsby PJ. Visual snow: persistent positive visual phenomenon distinct from migraine aura. Curr Pain Headache Rep 2015;19:23.

2. Schankin CJ, Maniyar FH, Digre KB, Goadsby PJ. Visual snow: a disorder distinct from persistent migraine aura. Brain 2014;137:1419-1428.

3. Battista J, Badcock DR, McKendrick AM. Migraine increases centre-surround suppression for drifting visual stimuli. PLoS One 2011;6:e18211.

4. McKendrick AM, Battista J, Snyder JS, Carter OL. Visual and auditory perceptual rivalry in migraine. Cephalalgia 2011;31:1158-1169.

5. Shepherd AJ, Joly-Maschernoni RM. Visual motion processing in migraine: enhanced motion after-effects are related to display contrast, visual symptoms, visual triggers and attack frequency. Cephalalgia 2016:1-12.

6. Wagner D, Manahilov V, Gordon GE, Storch P. Long-range inhibitory mechanisms in the visual system are impaired in migraine sufferers. Cephalalgia 2012;32:1071-1075.

7. Tsai JJ, Norcia AM, Ales JM, Wade AR. Contrast gain control abnormalities in idiopathic generalised epilepsy. Ann Neurol 2011;70:574-582.

8. Wagner D, Manahilov V, Loffler G, Gordon GE, Dutton GN. Visual noise selectively degrades vision in migraine. Invest Ophthalmol Vis Sci 2010;51:2294-2299.

9. Webster KE, Dickinson JE, Battista J, McKendrick AM, Badcock DR. Evidence for increased internal noise in migraineurs for contrast and shape processing. Cephalalgia 2012;32:125-139.

10. Chubb C, Sperling G, Solomon JA. Texture interactions determine perceived contrast. Proc Natl Acad Sci 1989;86: 9631-9635.

11. Newsome WT, Britten KH, Movshon JA. Neuronal correlates of a perceptual decision. Nature 1989;341:52-54.

12. Newsome WT, Pare EB. A selective impairment of motion perception following lesions of the middle temporal visual area (MT). J Neurosci 1988;8:2201-2211.
13. Koyama S, Sasaki Y, Anderson GJ, Tootell RBH, Matsuura M, Watanabe T. Separate processing of different global-motion structures in visual cortex is revealed by fMRI. Curr Biol 2005; 15:2027-2032.

14. Smith MA, Bair W, Movshon JA. Signals in macaque striate cortical neurons that support the perception of glass patterns. J Neurosci 2002;22:8334-8345.

15. Wilson HR, Wilkinson F. Detection of global structure in glass patterns: implications for form vision. Vis Res 1998; 38:2933-2947.

16. Angelucci A, Bressloff PC. Contribution of feedforward, lateral and feedback connections to the classical receptive field center and extra-classical receptive field surround of primate V1 neurons. Prog Brain Res 2006;154:93-120.

17. McCulloch DL, Marmor MF, Brigell MG, et al. ISCEV Standard for full-field clinical electroretinography (2015 update). Doc Ophthalmol 2015;130:1-12.

18. Dakin S, Carlin P, Hemslsy D. Weak suppression of visual context in chronic schizophrenia. Curr Biol 2005;15:R822R824.

19. Nguyen BN, McKendrick AM. Foveal and parafoveal contrast suppression are different: mechanisms revealed by the study of healthy ageing. J Vis 2016;16:10.

20. Karas R, McKendrick AM. Aging alters surround modulation of perceived contrast. J Vis 2009;9:11-19.

21. Karas R, McKendrick AM. Contrast and stimulus duration dependence of perceptual surround suppression in older adults. Vis Res 2015;110:7-14.

22. Glass L. The Moire effect from random dots. Nature 1969; 223:578-580.

23. Bair W, Cavanaugh JR, Movshon JA. Time course and time-distance relationships for surround suppression in macaque V1 neurons. J Neurosci 2003;23:7690-7701.

24. Petrov Y, McKee SP. The time course of contrast masking reveals two distinct mechanisms of surround suppression. $\mathrm{J}$ Vis 2009;9:1-11.

25. Schallmo MP, Murray SO. Identifying separate components of surround suppression. J Vis 2016;16:1-12.

26. Nurminen L, Angelucci A. Multiple components of surround modulation in primary visual cortex: multiple neural circuits with multiple functions? Vis Res 2014; 104:47-56.

27. Shushruth S, Nurminen L, Bijanzadeh M, Ichida JM, Vanni S, Angelucci A. Different orientation tuning of near and far surround suppression in macaque primary visual cortex mirrors their tuning in human perception. J Neurosci 2013; 33:106-119.

28. Tibber MS, Kelly MG, Jansari A, Dakin SC, Shepherd AJ. An inability to exclude visual noise in migraine. Invest Ophthalmol Vis Sci 2014;55:2539-2546.

29. Ditchfield JA, McKendrick AM, Badcock DR. Processing of global form and motion in migraineurs. Vis Res 2006; 46:141-148.

30. Antal A, Temme J, Nitsche MA, Varga ET, Lang N, Paulus W. Altered motion perception in migraineurs: evidence for interictal cortical hyperexcitability. Cephalalgia 2005;25:788-794.

31. McKendrick AM, Badcock DR, Gurgone M. Vernier acuity is normal in migraine whereas global form and global motion perception is not. Invest Ophthalmol Vis Sci 2006;47:3213-3219.

32. McKendrick AM, Badcock DR, Badcock JC, Gurgone M. Motion perception in migraineurs: abnormalities are not related to attention. Cephalalgia 2006;26:1131-1136. 